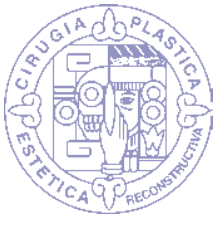

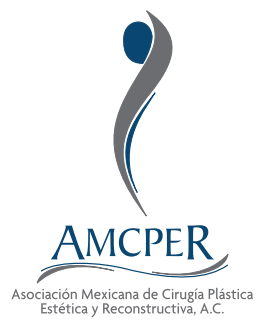

\title{
Estado nutricional y crecimiento en niños nacidos con fisuras labio palatinas
}

\author{
Nutritional status and growth in children born with cleft lip and palate \\ Dra. Rocío Gallego-Sobrino,* Dra. Leire Esparza-Lasaga,* Dr. Ignacio Moral-Sáez,* \\ Dr. Gerardo Rodríguez-Martínez ${ }^{\ddagger}$
}

Palabras clave: Fisura labio palatina, crecimiento, alimentación, nutrición, desnutrición.

Keywords: Cleft lip and palate, growth, feeding, nutrition, undernutrition.

* Médico Cirujano Maxilofacial. Servicio de Cirugía Oral y Maxilofacial, Hospital Universitario Miguel Servet. Zaragoza, España.

‡ Médico Pediatra (MD PhD). Área de Pediatría, Universidad de Zaragoza, Instituto de Investigación Sanitaria de Aragón. Red de Salud Materno-infantil y del Desarrollo (SAMID), RETICS, ISCIII, España.

Los autores de este artículo no tienen conflicto de intereses qué declarar.

Recibido:

24 febrero 2020 Aceptado para publicar: 06 marzo 2020

\section{RESUMEN}

En niños con fisura labio palatina se puede ver afectado el estado nutricional, el desarrollo y/o el crecimiento por las dificultades en la alimentación. En este artículo se revisa la literatura existente sobre el pronóstico nutricional de los niños nacidos con dicho defecto y la repercusión en su patrón de crecimiento. Estos pacientes pueden presentar un crecimiento disminuido en sus primeros meses de vida $\mathrm{y}$, debido al riesgo nutricional, necesitan inicialmente soporte y ayuda con la alimentación. Sin embargo, a partir de los dos años de edad, el pronóstico nutricional y el crecimiento se equiparán en la mayoría de ellos al de sus coetáneos. Se necesitan análisis más amplios que valoren el crecimiento de los niños con mayor riesgo nutricional y las consecuencias a largo plazo en su estado nutricional y en la composición corporal, tanto por defecto como por exceso.

\section{ABSTRACT}

In children with cleft lip and palate, nutritional status, development and/or growth can be affected because of feeding difficulties. This article reviews what has been written on the nutritional prognosis of children born with this defect and the impact on their growth pattern. These patients may have impaired growth in their first months of life and, due to nutritional risks; they need support initially and aid with feeding practices. However, after two years of age, the nutritional prognosis and growth in most of them are similar to their peers. More analyses are needed to assess the growth of those children with higher nutritional risk and the long-term consequences on their nutritional status and body composition both of default and excess.

\section{INTRODUCCIÓN}

L a fisura labio palatina congénita constituye ـ una deformidad relativamente común que produce, además del defecto estético estigmatizante, problemas funcionales relevantes en la deglución, la masticación, la fonación y en el crecimiento facial. Son anomalías congénitas que afectan a uno de cada $700-750$ recién nacidos, originadas por defectos embriológicos en la formación de la cara durante las primeras etapas del embarazo, por ausencia de fusión de los procesos maxilares y nasales en el caso de la fisura labial, o de los procesos palatinos en la fisura palatina. ${ }^{1}$

Las fisuras labio palatinas pueden clasificarse de varias formas en función de diversos criterios: embriológico, anatómico, odontológico y quirúrgico. La etiología se explica por una interacción entre factores genéticos y ambientales no bien definidos en la mayoría de los casos. Las consecuencias más frecuentes son problemas en la alimentación y en la respiración, infecciones del oído y pérdida auditiva, patología del habla, problemas dentarios y de desarrollo de los maxilares. El manejo de las fisuras labio palatinas requiere un tratamiento multidisciplinar (quirúrgico, ortopédico, ortodóncico, otorrinolaringológico, foniátrico y psicológico) que debe hacerse precozmente para evitar las alteraciones funcionales y posibilitar un desarrollo normal del niño, ${ }^{2}$ aunque puede requerir tratamiento durante la adolescencia e, incluso, en etapa adulta. 
El estado nutricional, el desarrollo y el crecimiento de los niños con fisura labio palatina se pueden ver afectados por la dificultad para alimentarlos de manera adecuada, debido principalmente a su anatomía y a las propias intervenciones quirúrgicas. Además, de manera secundaria, los procesos infecciosos de las vías aéreas superiores o del oído medio son otros factores que pueden contribuir a esta alteración nutricional. ${ }^{3,4}$

El objetivo del presente artículo es revisar la literatura existente sobre el pronóstico nutricional de los pacientes intervenidos de fisura labio palatina, las peculiaridades en su composición corporal y en su patrón de crecimiento así como los posibles efectos de los problemas de alimentación de estos niños en la aparición de sobrepeso y obesidad a largo plazo.

\section{PROBLEMAS DE ALIMENTACIÓN ASOCIADOS A LA FISURA LABIO PALATINA}

Los pacientes fisurados presentan una comorbilidad que requiere manejo multidisciplinar. El equipo de profesionales recomendado por la American Cleft Palate Association (ACPA) y el Eurocleft debe estar compuesto por cirujano maxilofacial, ortodoncista, logopeda, otorrinolaringólogo, pediatra, odontopediatra, genetista/dismorfólogo y psicólogo, entre otros. ${ }^{5}$ La interacción entre los componentes del equipo permitirá el manejo integral de la deformidad para asegurar el mejor resultado posible.

La presencia de estas malformaciones congénitas puede tener efecto negativo en el crecimiento y desarrollo durante la infancia. Las fisuras labio palatinas afectan funciones importantes (sistema respiratorio y digestivo) que intervienen directa y decisivamente en el estado nutricional. Además, dependiendo del tipo de fisura que presente el lactante, se va a alterar la habilidad de éste para alimentarse de manera adecuada. Los pacientes con fisuras que afectan sólo el labio, o labio y reborde alveolar, no deberían tener problemas para alimentarse directamente del pecho materno. ${ }^{6}$ Los niños con fisuras labiales completas unilaterales o bilaterales, así como con fisura de paladar presentarán con más frecuencia dificultades en la alimentación, fundamentalmente debido a que el labio leporino puede comprometer la succión durante la lactancia y el paladar hendido puede provocar que la leche pase a la cavidad nasal. ${ }^{3,4,6}$

En términos anatómicos, un paladar abierto puede afectar la capacidad de un lactante para alimentarse de varias maneras. En primer lugar, puede tener un efecto en la mecánica bucal y en la capacidad de generar la presión intraoral negativa necesaria para una succión efectiva. Además, puede provocar dificultad en la coordinación de la succión, la deglución y la respiración durante la alimentación, aumentando el riesgo de broncoaspiración. ${ }^{7}$ Los pacientes no sindrómicos con fisura labial y palatina unilateral completa o una fisura de paladar blando y al menos dos tercios del paladar duro muestran patrones de succión menos eficientes que sus coetáneos sanos. Estos pacientes utilizan succiones más cortas, una velocidad de succión más rápida, mayor tasa de succión-deglución y generan mayor presión positiva intraoral. ${ }^{7}$

En general, cuanto más extensa es la fisura del paladar, mayor es la posibilidad de presentar problemas de alimentación y una ingesta deficiente. Se deben identificar dichos problemas y aplicar tratamiento de manera precoz, ya que es necesario conseguir un volumen adecuado de ingesta y un peso óptimo antes de la reparación quirúrgica de la fisura. ${ }^{8}$ Además, los tiempos prolongados de ingesta pueden ser muy estresantes, tanto para el lactante como para los padres, lo que provoca rechazo y alteración del vínculo placentero que normalmente se genera con la alimentación. La mayoría de los lactantes sanos pueden completar una ingesta por succión en 20-30 minutos; sin embargo, el paciente con fisura palatina por lo regular tarda mucho más en alimentarse. Las asociaciones científicas y las de familias de pacientes afectos disponen de recursos en internet para padres y cuidadores sobre cómo alimentar a un lactante con una fisura. ${ }^{5}$

La adecuada alimentación en los pacientes con fisura labio palatina es una prioridad y constituye el desafío más inmediato al que se enfrentan los padres después del nacimiento. ${ }^{9}$ De hecho, los padres afirman que la obtención de las instrucciones adecuadas sobre los méto- 
dos efectivos de alimentación es una prioridad durante las primeras semanas. Existen múltiples dispositivos para ayudar a la alimentación en estos pacientes: chupete asociado a jeringa, biberones con una aleta tipo obturador para cerrar la comunicación palatina o biberones blandos que se aprietan facilitando la ingesta debido al fallo del sistema de succión presente en estos niños. ${ }^{10}$

La primera opción alimenticia la constituye, como para cualquier niño, la lactancia materna. De no ser posible esta opción, dadas las dificultades de succión, se podrán utilizar sistemas como los anteriormente citados para la ingesta de leche humana o de fórmula, evitando en lo posible cualquier sistema de nutrición «pasivo» como la sonda nasogástrica, a la que sólo se recurrirá como última opción. Se intentará mantener al niño en una posición vertical, evitando en lo posible la posición horizontal que aumenta la probabilidad de regurgitación nasal, tos, estornudos, así como fenómenos de aspiración. ${ }^{10}$ Además, puede haber paso de contenido alimenticio a la trompa de Eustaquio y reflujo en el oído medio. Una posición semivertical (de al menos 60 grados) es mejor para la alimentación, ya que facilita el control de los movimientos de la mandíbula, las mejillas, los labios y la lengua para la coordinación de la succión y la deglución. ${ }^{5}$

Una buena intervención para el manejo de la alimentación incluye una mejora en los aspectos de comunicación dentro del equipo multidisciplinar con la incorporación de asistente social, seguimiento ambulatorio de los pacientes y educación del personal así como un buen acceso al equipo de nutrición con experiencia en alimentación en casos de fisura palatina. ${ }^{11}$ Con un programa de intervención que contemplaba estos aspectos, Baylis y colaboradores ${ }^{11}$ mostraron que la tasa de fallo de medro en los lactantes con fisura labio palatina disminuía de 17 a $7 \%(p<0.003)$ y la frecuencia de hospitalización por dificultades en la alimentación mejoraba. Las intervenciones dirigidas a implementar la alimentación, así como los cambios en los modelos de prestación de atención, hacen que los resultados nutricionales y el crecimiento de los lactantes con fisura labio palatina mejoren, incluso cuando existen factores de riesgo psicosocial. ${ }^{11}$
Se deben tener en cuenta varias recomendaciones para alimentar a los lactantes con fisura labio palatina, tales como evaluar la capacidad de succión, enseñar la posición adecuada para la lactancia materna, ayudar a la familia a establecer y mantener el suministro de leche, un equipo de alimentación adaptable (biberones y tetinas específicos) y educación sobre aspectos de nutrición infantil. El apoyo de los profesionales de la salud, en particular los especialistas en lactancia y el personal de enfermería, es fundamental. ${ }^{12}$ Estos pacientes tienen limitaciones físicas específicas y se necesitan medidas especiales para asegurar el soporte nutricional que permita un adecuado crecimiento y la mejor de las situaciones antes de una intervención quirúrgica. La incorporación de prótesis obturadoras también puede ayudar en el manejo de la regurgitación nasal y la aspiración de alimento por la imposibilidad del paladar fisurado para separar las cavidades nasal y oral. ${ }^{13}$

En cuanto a las recomendaciones de alimentación después de la reparación quirúrgica de la fisura labial y/o palatina varían entre los diferentes centros y sigue siendo un tema controvertido. Algunos grupos permiten la alimentación inmediata sin restricciones, mientras que otros recomiendan un enfoque más progresivo para facilitar una buena curación. Por ejemplo, algunos centros desaconsejan la succión después de la cirugía de la fisura labial y/o palatina y recomiendan el uso de una taza o una cuchara en su lugar, incluso en ocasiones prefieren la alimentación inicial con sonda nasogástrica durante un periodo de siete a 10 días. En contraste, algunos centros han instaurado la alimentación inmediata mediante succión o cuchara después de la reparación de la fisura sin problemas. ${ }^{14}$

\section{ALTERACIONES NUTRICIONALES ASOCIADAS}

La desnutrición por malnutrición secundaria se asocia a un gran número de enfermedades en nuestro medio, entre las que destacan las malformaciones congénitas, las afecciones crónicas respiratorias y digestivas, los trastornos del metabolismo, cáncer, etcétera. ${ }^{15}$

Los factores que causan alteración nutricional en niños con malformación labio pala- 
tina son las dificultades de alimentación y de ingestión durante los primeros meses de vida, las intervenciones quirúrgicas y sus secuelas así como los procesos infecciosos en las vías aéreas o en el oído medio. ${ }^{16}$ Sin embargo, algunos estudios que han comparado el estado nutricional de pacientes con diferentes tipos de fisuras no han observado diferencias significativas entre los grupos, o entre éstos y sus coetáneos nacidos sin fisuras. ${ }^{17,18}$ Se ha comprobado cómo estos pacientes tienen un potencial de crecimiento genético normal en su mayoría y es importante asegurar un adecuado soporte familiar y nutricional. ${ }^{3,4,7}$ En estudios con poblaciones homogéneas y comparables se ha mostrado que los pacientes con fisura labio palatina aislada, sin síndromes genéticos asociados, tienen un patrón de crecimiento adecuado según los estándares de referencia. ${ }^{19}$ Así pues, las desviaciones de dichas trayectorias de crecimiento deben considerarse anormales de igual manera que se haría en un niño sin fisuras. Si además de la fisura existe comorbilidad asociada, el crecimiento se puede ver afectado por causas intrínsecas y ajenas a la fisura.

Otro aspecto que se ha investigado es si ya existen diferencias en el peso de recién nacidos con fisura labial y/o palatina respecto a los sanos. Los resultados varían y no son concluyentes, pero en algún caso sugieren que estos pacientes tienen menor peso al nacer $y$ son más pequeños que los nacidos sin fisura. La variación en los resultados puede deberse a factores de confusión tales como la edad materna, la paridad $\mathrm{u}$ otros factores sociales. ${ }^{20,21}$ Este punto debe tenerse en cuenta a la hora de evaluar efectos a largo plazo de la nutrición en pacientes fisurados y su relación con la «programación» del crecimiento tardío.

A este respecto, Kaye y colaboradores ${ }^{22}$ en un estudio reciente evaluaron longitudinalmente a 100 pacientes recién nacidos con diagnóstico de fisura labial y/o fisura palatina entre 2009 y 2012, dividiendo la muestra en tres grupos: fisura labial, fisura palatina y fisura labio palatina. El peso promedio al nacer fue similar en los tres grupos. La edad para la recuperación del peso al nacer fue significativamente diferente: 13.5 días en la fisura labial; 15.88 días en la fisura labio palatina y 21.93 días en la fisura palatina. Otro aspecto importante analizado en este estudio fue el uso de lactancia materna exclusiva que ocurría en $50 \%$ del grupo de fisura labial, en $30.3 \%$ del de fisura labio palatina y en $21.4 \%$ del de fisura palatina. Además, en el grupo de fisura labial hubo un aumento de peso significativamente mayor en su primera visita y requirió menos intervenciones nutricionales que los grupos de fisura palatina y fisura labio palatina. ${ }^{22} \mathrm{La}$ lactancia materna y el uso de leche humana deben ser priorizados y respaldados.

En un estudio similar en el año 2005, Montagnoli y su equipo ${ }^{21}$ analizaron las diferencias en la restricción de crecimiento hasta el segundo año de edad, dividiendo igualmente la muestra en tres grupos: fisura labial, fisura palatina y fisura labio palatina. Se obtuvieron mediciones de peso y longitud y se analizó la alimentación mediante lactancia materna exclusiva. En este estudio, la lactancia materna exclusiva también fue más frecuente en el grupo de fisura labial $(45.9 \%)$ que en la fisura palatina $(12.1 \%)$ o en la fisura labio palatina (10.5\%). Los niños con fisura labial mostraron un deterioro menos marcado del peso $(23.8 \%)$ y la longitud (19.3\%) en comparación con el grupo de fisura palatina y fisura labio palatina (35.7 y $33.1 \%$, respectivamente). En estos dos últimos grupos, la proporción de niños con peso y longitud por debajo del percentil 10 fue muy similar (34.4 y $38.9 \%$ ). Estos resultados se pueden atribuir a la mayor dificultad en la alimentación en los pacientes que asocian fisura palatina en comparación con el grupo de fisura labial aislada. ${ }^{21}$

Miranda y $\operatorname{colegas}^{20}$ estudiaron la evolución de la longitud, del peso y del índice de masa corporal (IMC) en pacientes menores de dos años con fisura labial y fisura palatina, con dieta normal, con el objetivo de establecer curvas de crecimiento específicas para estos niños. Para la comparación con sus coetáneos sanos se utilizaron como referencia las tablas de crecimiento de la Organización Mundial de la Salud (OMS) de 2006. ${ }^{23}$ En los resultados se observó que los pacientes con fisura labio palatina y fisura palatina tenían un peso e IMC por debajo de las curvas de la OMS, pero mostraron una recuperación espontánea a partir de los cinco meses de edad. Los pacientes con fisura labial asilada tuvieron un crecimiento similar 
al de los niños sanos desde el principio. Los pacientes con fisura labio palatina y palatina que inicialmente tenían valores de peso e IMC menores que los del grupo de fisura labial aislada, mostraban después de los nueve meses de edad un peso e IMC igual o mayor que el grupo de fisura labial aislada. ${ }^{20}$

Según el estudio anterior, los pacientes con más afectación nutricional durante los primeros cinco meses de edad podrían presentar más tarde un aumento de peso rápido y compensador. Si se revisa el resto de la literatura, pese a las diferencias que se evidencian durante los primeros meses, el estado nutricional de la mayoría de estos pacientes podría equipararse al de sus coetáneos sanos antes del año de edad, ${ }^{17,20}$ aunque hay un estudio que muestra diferencias que se mantienen hasta los dos años de edad. ${ }^{21}$

\section{CRECIMIENTO Y COMPOSICIÓN CORPORAL A LARGO PLAZO}

La restricción del crecimiento por desnutrición intraútero y/o postnatal que se mantiene en el tiempo puede disminuir el potencial de crecimiento y estado nutricional posterior, programando al niño hacia la delgadez, menor talla final y menor cantidad de masa muscular. ${ }^{24}$ Por otro lado, se ha comprobado que la desnutrición temprana con periodos de crecimiento recuperador rápido, programa un crecimiento excesivo y mayor riesgo de adiposidad a largo plazo. ${ }^{24,25}$ Como se ha visto en el apartado anterior, los pacientes con fisura labio palatina pueden tener riesgo de desnutrición durante los dos primeros años de edad, ${ }^{21,22}$ o de crecimiento recuperador rápido, ${ }^{20}$ con las consecuencias que todo ello conlleva. Los estudios disponibles sobre el crecimiento y estado nutricional a largo plazo de niños con fisura labio palatina son escasos en la literatura.

A este respecto, Marques y colaboradores ${ }^{19}$ midieron en un estudio longitudinal la estatura y el peso de 360 pacientes con fisura labio palatina completa unilateral aislada, sin síndromes asociados, de dos a 10 años de edad. Para comparar el crecimiento de los mismos con el de sus coetáneos sanos se desarrollaron curvas de crecimiento y se compararon con los patrones de referencia de la OMS de 2006. ${ }^{23}$
En este estudio, los pacientes con fisura labio palatina completa unilateral de dos a 10 años presentaron curvas de crecimiento en altura y peso similares a las de los niños sanos en ambos sexos. ${ }^{19}$

Al revisar con más detalle qué ocurre con el crecimiento durante los dos primeros años de edad los resultados son controvertidos. Por un lado existen estudios clásicos en los que al final de este periodo hay un aumento considerable de niños con fisura palatina o labio palatina con peso y talla por debajo del P10. ${ }^{21}$ En un estudio más reciente en línea del trabajo de Marques y su equipo ${ }^{19}$ y con datos sobre alimentación se muestra cómo el peso y longitud en pacientes con fisura labio palatina completa unilateral aislada fueron idénticos a los valores de referencia de la OMS tanto al nacer como a los seis, 12,18 y 24 meses de edad. ${ }^{17}$ Miranda y colegas $^{20}$ incluso llegaron a comprobar un incremento del IMC en algunos pacientes con fisura labio palatina y palatina al final del primer año de edad frente a sus coetáneos con fisura labial aislada.

Respecto a la alimentación y su relación con el crecimiento, Gopinath ${ }^{18}$ revisó la ingesta de nutrientes en un total de 139 pacientes de ambos sexos, de dos a seis años de edad con fisura labial y/o fisura palatina, la comparó con un grupo control y evidenció que no había diferencias significativas entre ambos grupos. ${ }^{18}$ Así pues, el crecimiento a largo plazo se iguala cuando la ingesta es similar entre niños con fisura y sus coetáneos sanos.

Koltz y colaboradores ${ }^{26}$ analizaron a largo plazo el IMC de pacientes con fisura labial y/o palatina aislada utilizando puntajes z-score de IMC ajustados por edad. Se obtuvieron al menos dos medidas de altura y peso durante dos a 10 años y al menos dos medidas de altura y peso durante 10-20 años. Si bien las diferencias no eran significativas, los resultados globales sugerían que la población con antecedentes de fisura labio palatina mantiene un IMC medio similar al de la población general durante la infancia y la adolescencia. ${ }^{26}$

\section{COMENTARIOS FINALES}

Con esta revisión y a modo de resumen, existe suficiente evidencia para afirmar que los pa- 
cientes con fisuras labiales y/o palatinas pueden tener un crecimiento disminuido en sus primeros meses de vida y representan inicialmente un grupo de riesgo nutricional. Dichos pacientes necesitan soporte nutricional y ayuda con la alimentación durante la primera infancia. Sin embargo, a largo plazo, el pronóstico nutricional y el crecimiento se equiparan en la mayoría de ellos al de sus coetáneos, sobre todo a partir de los dos años de edad. En términos generales, los niños con afectación palatina tienen más problemas de alimentación y nutricionales que aquéllos con afectación sólo labial; además, si existe patología asociada o sindrómica, el pronóstico en la trayectoria de crecimiento empeora. Se necesitan análisis más amplios que valoren el crecimiento de los niños con mayor riesgo nutricional y las consecuencias a largo plazo en su estado nutricional y en la composición corporal tanto por defecto como por exceso y en diferentes compartimentos corporales.

\section{REFERENCIAS}

1. Vázquez-Mahía I, Patiño-Seijas B. Fisura labio palatina. En: Sociedad Española de Cirugía Oral y Maxilofacial (SECOM). Manual de cirugía oral y maxilofacial. 2. a ed. Madrid: SECOM; 2004. pp. 1347-1379.

2. González G, Prado MC. Guía de las fisuras labio palatinas. Una patología crónica. ASPANIF; 2011. ISBN 978-84-6952679-8.

3. Glenny AM, Hooper L, Shaw WC, Reilly S, Kasem $S$, Reid J. Feeding interventions for growth and development in infants with cleft lip, cleft palate or cleft lip and palate. Cochrane Database Syst Rev 2004; (3): CD003315.

4. Bessell A, Hooper L, Shaw WC, Reilly S, Reid J, Glenny AM. Feeding interventions for growth and development in infants with cleft lip, cleft palate or cleft lip and palate. Cochrane Database Syst Rev 2011; (2): CD003315.

5. American Cleft Palate-Craniofacial Association. Parameters for evaluation and treatment of patients with cleft lip/ palate or other craniofacial anomalies. Revised Edition 2009. Cleft Palate Craniofac J 2018; 55: 137-156.

6. Ranganathan K, Vercler CJ, Warschausky SA, MacEachern MP, Buchman SR, Waljee JF. Comparative effectiveness studies examining patient-reported outcomes among children with cleft lip and/or palate: a systematic review. Plast Reconstr Surg 2015; 135: 198-211.

7. Masarei AG, Sell D, Habel A, Mars M, Sommerlad BC, Wade A. The nature of feeding in infants with unrepaired cleft lip and/or palate compared with healthy non cleft infants. Cleft Palate Craniofac J 2007; 44: 321-328.
8. Kummer AW. Cleft palate and craniofacial anomalies: effects on speech and resonance. 3rd ed. Clifton Park, NY: Cengage Learning; 2014. pp. 131-164.

9. Shaw WC, Semb G, Nelson P, Brattstrom V, Molsted K, Prahl-Andersen B et al. The Eurocleft project 19962000: overview. J Cranio-Maxillofacial Surg 2001; 29 (3): 131-140.

10. Kim EK. Effect of unrestricted bottle-feeding on early postoperative course after cleft palate repair. J Craniofac Surg 2009; 20: 1886-1888.

11. Baylis AL, Pearson GD, Hall C, Madhoun LL, Cummings $\mathrm{C}$, Neal $\mathrm{N}$ et al. A quality improvement initiative to improve feeding and growth of infants with cleft lip and/or palate. Cleft Palate Craniofac J 2018; 55: 12181224.

12. Burca ND, Gephart SM, Miller C, Cote C. Promoting breast milk nutrition in infants with cleft lip and/or palate. Adv Neonatal Care 2016; 16: 337-344.

13. Goswami M, Jangra B, Bhushan U. Management of feeding problem in a patient with cleft lip/ palate. Int J Clin Pediatr Dent 2016; 9: 143-145.

14. Katzel E. Current surgical practices in cleft care: cleft palate repair techniques and postoperative care. Plast Reconstr Surg 2009; 124: 899-906.

15. Moreno LA, Rodríguez G. Valoración del estado nutricional. En: Moro M, Málaga S, Madero L, eds. Tratado de pediatría cruz. 11 1 a ed. Madrid: Panamericana; 2014. pp. 1031-1038.

16. Holland S. Delayed closure of the hard palate leads to speech problems and deleterious maxillary growth. Plast Reconstr Surg 2007; 119: 1302-1310.

17. McHeik JN, Levard G. Growth in infants in the first two years of life after neonatal repair for unilateral cleft lip and palate. Int J Pediatr Otorhinolaryngol 2010; 74: 465-468.

18. Gopinath VK. Assessment of nutrient intake in cleft lip and palate children after surgical correction. Malays J Med Sci 2013; 20: 61-66.

19. Marques IL, Nackashi J, Borgo HC, Martinelli AP, de Souza L, Dutka Jde C et al. Longitudinal study of growth of children with unilateral cleft lip and palate: 2 to 10 years of age. Cleft Palate Craniofac J 2015; 52: 192-197.

20. Miranda GS, Marques IL, de Barros SP, Arena EP, de Souza L. Weight, length, and body mass index growth of children under 2 years of age with cleft lip and palate. Cleft Palate Craniofac J 2016; 53: 264-271.

21. Montagnoli LC, Barbieri MA, Bettiol H, Marques IL, de Souza L. Growth impairment of children with different types of lip and palate clefts in the first 2 years of life: a cross-sectional study. J Pediatr (Rio J) 2005; 81: 461-465.

22. Kaye A, Thaete K, Snell A, Chesser C, Goldak C, Huff $\mathrm{H}$. Initial nutritional assessment of infants with cleft lip and/or palate: interventions and return to birth weight. Cleft Palate Craniofac J 2017; 54: 127-136.

23. WHO child growth standards: methods and development: length/height-for-age, weight-for-age, weight-for-length, weight-for-height, body mass index-for-age. Geneva: World Health Organization; 2006. Disponible en: http://www.who.int/childgrowth/ publications.

24. Biosca M, Rodríguez G, Ventura P, Samper MP, Labayen I, Collado MP et al. Central adiposity in children born 
small and large for gestational age. Nutr Hosp 2011; 26: 971-976.

25. Iguacel I, Escartín L, Fernández-Alvira JM, Iglesia I, Labayen I, Moreno LA et al. Early-life risk factors and their cumulative effects as predictors of overweight in Spanish children. Int J Public Health 2018; 63: 501-512.

26. Koltz PF, Wasicek P, Mays C, Bloom J, Girotto JA. Growth trajectory of children and adolescents with isolated cleft lip and/or palate through the first two decades of life. Int J Oral Maxillofac Surg 2012; 41: 1244-1247.

Correspondencia:

Dra. Rocío Gallego-Sobrino

Hospital Universitario Miguel Servet.

Paseo de Isabel La Católica 1-3,

50009, Zaragoza, España.

E-mail: ronchi_g@hotmail.com 